\title{
Product-state distribution after isotopic substitution in ultracold atom-molecule collisions
}

\author{
Maciej B. Kosicki, Piotr S. Żuchowski \\ E-mail: pzuch@fizyka.umk.pl \\ Institute of Physics, Faculty of Physics, Astronomy and Informatics, Nicolaus \\ Copernicus University, Grudziadzka 5, 87-100 Toruń, Poland
}

\author{
Maykel L. González-Martínez and Olivier Dulieu \\ E-mail: olivier.dulieu@u-psud.fr \\ Laboratoire Aimé Cotton, CNRS, Université Paris-Sud, ENS Paris-Saclay, Université \\ Paris-Saclay, \\ Bât. 505, Campus dOrsay, 91405 Orsay, France
}

11 October 2019

\begin{abstract}
We show that products of the isotopic substitution reactions in experimentally accessible molecules such as $\mathrm{NaK}$, RbCs and $\mathrm{SrF}$ are cold according to their translational energy below hundreds of mK. For these chemical reactions molecular products may occupy only the lowest rotational states. We also discuss the possibility of controlling the chemical reactions by the electric field in ultracold mixtures of molecules and atoms with low kinetic energy release, where one of the constituent atoms of colliding molecule is replaced by its isotope. This letter open new avenues in investigating the branching ratios of chemical reactions in ultracold conditions.
\end{abstract}

Submitted to: J. Phys. B: At. Mol. Opt. Phys. 
The past decade has witnessed rapid progress in the investigation of chemistry at ultracold temperatures. Ospelkaus et al. [1] demonstrated field-free chemical reactions between KRb molecules and constituent atoms. By controlling the internal state of KRb molecule, it was possible to enhance the reaction rate between two KRb molecules by allowing $s$-wave collisions. They have also described collisions of KRb with ultracold $\mathrm{K}$ or $\mathrm{Rb}$ atoms finding that a formation of $\mathrm{K}+\mathrm{Rb}_{2}$ products is energetically forbidden. Later on, the same experimental group [2, 3] has also shown that an external electric field may be applied to orient KRb molecules and to influence their reaction rate. Recently, a mechanism to monitor the chemical reactivity by the selection of reactant vibrational state has been utilized in the reaction between $\mathrm{NaRb}$ molecules [4]. Other experimental attempts involve Feshbach molecules and ultracold atoms in the presence of an external magnetic field [5, 6].

Measurements of the reactant loss is a straightforward way to probe reactivity at ultracold temperatures. However, the detection of products would provide much more details about the underlying dynamics. A basic difficulty in detecting product states of such reactions is that the kinetic energy release is often larger by several orders of magnitude than the depths of most traps used in experiments (see e.g. [7, 8]). Despite several successful realizations of ultracold molecular gases of ground-state heteronuclear alkali-metal diatomic species [9, 10, 11, 12, 13, 14, 15, 16, 17] and promising prospects [18, 19], no detailed study of product-state distribution has yet been reported so far. However two important steps in this direction were recently achieved: the detection of weakly-bound ${ }^{87} \mathrm{Rb}_{2}$ molecules by three-Rb-atom recombination [20, 21], and the detection of polyatomic ions after the collision of two ultracold KRb molecules [22].

It would be desirable to explore cases in which the products can be accumulated in the trap after the chemical reaction. Inspired by a recent proposal for chemical reaction with very small energy release, involving isotope exchange in ultracold molecular collisions [23], we investigate in the present work the isotopic substitution reactions between a ground-state alkali-metal atom ${ }^{a} \mathrm{~A}$ with atomic mass $a$ and a ground-state heteronuclear diatomic molecule containing a different isotopic species ${ }^{b} \mathrm{~A}$ (with $a>b$ ), and another atom B

$$
{ }^{a} \mathrm{~A}+{ }^{b} \mathrm{AB} \longrightarrow{ }^{b} \mathrm{~A}+{ }^{a} \mathrm{AB} .
$$

If the molecule is initially in its lowest rovibrational level $v=0, n=0$, (neglecting the hyperfine structure for now), the energy release $\Delta E_{a-b}$ for the reaction (1) can be expressed in terms of the change of the zero-point energy

$$
\Delta E_{a-b}=\frac{\omega_{a}-\omega_{b}}{2},
$$

where $\omega_{a}\left(\omega_{b}\right)$ is the harmonic constant of the ${ }^{a} \mathrm{AB}\left({ }^{b} \mathrm{AB}\right)$ molecule. Values of $\Delta E_{a-b}$ for a selected series of species of experimental interest are found small enough to be considered in future experiments based for instance on electrostatic or microwave traps (Table 1).

Our previous studies show that atom-exchange reactions involving alkali-metal dimers and atoms, as well as strontium monofluoride and strontium atoms, are 
barrierless processes [7, 8]. In all these cases the potential energy surfaces (PES) are very deep and support tens of vibrational levels and hundreds of rotational levels. Full quantum scattering calculations for atom-molecule reactive collisions, even for the field-free case, are cumbersome for strongly interacting species as alkali-metal atoms [24, 25, 26, 27, 28], due to the huge number of involved channels. Interestingly enough, the isotope-exchange of lithium in field-free atom-diatom collisions has been investigated in [26], and the product-state distribution for this system has been analyzed.

Recently, it was demonstrated [29, 30] that the distribution of product rotational states after the atom-exchange reaction between ultracold $\mathrm{K}$ atom and KRb molecule, calculated with rigorous quantum scattering calculations exhibits the Poissonian distribution, while the of positions and widths of scattering resonances display signatures of quantum chaos: Wigner-Dyson distribution of resonances spacings in energy domain, and Porter-Thomas distribution of widths of resonances. A similar conclusion has been reached for the $\mathrm{D}^{+}+\mathrm{H}_{2} \rightarrow \mathrm{H}^{+}+\mathrm{DH}$ reaction [31, 32. Furthermore, it was noticed in [33] that the collisions of alkali-metal dimers with atoms exhibit strongly resonant character: near the collision threshold, the density of bound states supported by closed channels (related to excited hyperfine, rotational and vibrational states of products) is very high. Because of that, long-lived collision complexes are predicted to be formed, and full randomization of the reaction energy (via internal modes) should occur before the complex is destroyed. This essentially makes the product state distribution of the complex statistical, i.e. without featuring correlation between products and reactants. We then use the statistical approach adopted in [34 for ultracold molecular collisions to investigate such isotope-substitution reactions when all particles are in their absolute ground state in free space. We will show how the electric field can be used to control their reactivities by blocking the reaction channels and by tuning product state distributions. Although we focus here on the electric field control, one could imagine a similar scenario with the magnetic field for paramagnetic molecules or microwave radiation fields.

For the chemical reactions addressed in Table 1, the kinetic energy release is small. Values for $\Delta E_{a-b}$ values have been obtained using experimental data [35, 36, 37, 38, 39, 40, 41] and mass-scaled harmonic frequencies of the ground state. Non-BornOppenheimer (NBO) effects, leading to an estimated shift of about dozens of $\mathrm{MHz}$ between the two isotopologues ${ }^{a} \mathrm{AB}$ and ${ }^{b} \mathrm{AB}$ for alkali-metal dimers [42], are neglected, so that they have both the same dissociation limit before including hyperfine structure. The same origin is considered for the hyperfine manifold of the colliding atomic partner. The values of $\Delta E_{a-b}$ are obtained after setting the origin of energies at this dissociation limit, which is the center of gravity of the hyperfine manifold of the constituent atoms. The hyperfine structure of the $(v=0, n=0)$ ground-state level is also neglected for these evaluations. The ${ }^{88} \mathrm{Sr}+{ }^{86} \mathrm{Sr}^{19} \mathrm{~F}$ reaction must be considered with care, as $\Delta E_{a-b}$ is only about $300 \mathrm{MHz}$ larger than the $n=0 \rightarrow n=1$ rotational excitation in ${ }^{88} \mathrm{Sr}^{19} \mathrm{~F}$. Using the same methodology as in 42, based on ADF quantum-chemistry code [43] with the density functional method (PBE96) [44], we found that the NBO shift between the ground-state $(v=0, n=0)$ level energies of ${ }^{88} \mathrm{Sr}^{19} \mathrm{~F}$ and ${ }^{86} \mathrm{Sr}^{19} \mathrm{~F}$, is smaller than 
$10 \mathrm{MHz}$, and thus, can be safely neglected.

Table 1. The calculated reaction energy $\Delta E_{a-b}$ (references to used data in square brackets), and the rotational constant $B_{0}$, the hyperfine coupling constant $A$ and spinorbit coupling constant $\gamma_{S R}$ of products are shown. Symbols $n_{\max }$ and $\ell_{\max }$ denote the maximum rotational state of molecule for a collision energy of $1 \mu \mathrm{K}$ and the maximum end-over-end angular momentum which can be populated by $\Delta E_{a-b}$, respectively. See supplementary material for complete compilation for all possible alkali-metal dimers reactions.

\begin{tabular}{lllllll}
\hline Products & $\Delta E_{a-b}[\mathrm{mK}]$ & $B_{0} / k_{b} T[\mathrm{mK}]$ & $A / k_{b} T[\mathrm{mK}]$ & $\gamma_{S R}[\mathrm{mK}]$ & $n_{\max }$ & $\ell_{\max }$ \\
\hline${ }^{39} \mathrm{~K}+{ }^{23} \mathrm{Na}^{40} \mathrm{~K}$ & $415[36]$ & $136[45]$ & $11[46]$ & - & 1 & 18 \\
${ }^{39} \mathrm{~K}+{ }^{23} \mathrm{Na}^{41} \mathrm{~K}$ & $811[36]$ & $136[45]$ & $11[46]$ & - & 1 & 23 \\
${ }^{40} \mathrm{~K}+{ }^{23} \mathrm{Na}^{41} \mathrm{~K}$ & $396[36]$ & $136[45]$ & $-14[46]$ & - & 1 & 18 \\
${ }^{39} \mathrm{~K}+{ }^{40} \mathrm{~K}^{87} \mathrm{Rb}$ & $472[37]$ & $55[47]$ & $11[46]$ & - & 2 & 22 \\
${ }^{39} \mathrm{~K}+{ }^{40} \mathrm{~K}^{133} \mathrm{Cs}$ & $478[38]$ & $44[48]$ & $11[46]$ & - & 2 & 23 \\
${ }^{85} \mathrm{Rb}+{ }^{7} \mathrm{Li}^{87} \mathrm{Rb}$ & $123[35]$ & $316[48]$ & $49[49]$ & - & 0 & 16 \\
${ }^{85} \mathrm{Rb}+{ }^{23}{ }^{2} \mathrm{Na}^{87} \mathrm{Rb}$ & $188[39]$ & $100[11]$ & $49[49]$ & - & 0 & 20 \\
${ }^{85} \mathrm{Rb}+{ }^{39} \mathrm{~K}^{87} \mathrm{Rb}$ & $198[37]$ & $55[47]$ & $49[49]$ & - & 1 & 22 \\
${ }^{85} \mathrm{Rb}+{ }^{87} \mathrm{Rb}^{133} \mathrm{Cs}$ & $253[40]$ & $23[50]$ & $49[49]$ & - & 1 & 27 \\
${ }^{86} \mathrm{Sr}+{ }^{88} \mathrm{Sr}^{19} \mathrm{~F}$ & $745[41]$ & $365[41]$ & $5[50]$ & $4[50]$ & 1 & 27 \\
\hline
\end{tabular}

The $\Delta E_{a-b}$ values displayed in Table1 range from $123 \mathrm{mk}$ to $811 \mathrm{mK}$, corresponding to the ${ }^{87} \mathrm{Rb}+{ }^{7} \mathrm{Li}^{85} \mathrm{Rb}$ and ${ }^{41} \mathrm{~K}+{ }^{23} \mathrm{Na}^{39} \mathrm{~K}$ collisions, respectively. These energy releases are larger than the typical depth of optical dipole traps, but it should be possible to retain the products in electrostatic traps or in microwave cavities, or even in magnetic traps for the case of monofluoride reactants. The maximal value $n_{\max }$ of the molecular rotational quantum number $n$ allowed by the energy release is deduced within the rigid-rotor approximation for the rotational energies $E(n)=n(n+1) B_{0}$, where $B_{0}$ is the rotational constant of the $(v=0, n=0)$ ground-state level. We neglected collision energy of $1 \mu \mathrm{K}$ in the $n_{\max }$ estimation. Interestingly, very few rotational levels can be populated in the products, and only $n=0$ in the cases of ${ }^{87} \mathrm{Rb}+{ }^{85} \mathrm{Rb}^{23} \mathrm{Na}$, and ${ }^{87} \mathrm{Rb}+{ }^{85} \mathrm{Rb}{ }^{7} \mathrm{Li}$ collisions. In contrast, very large number of hyperfine levels can be populated in the alkali-metal. Specifically, there are 144 hyperfine levels for both $n=0$ and $n=1$ rotational states of the $\mathrm{Na}^{40} \mathrm{~K}$ and even more for the ${ }^{87} \mathrm{RbCs}$ due to the presence of $n=2$ rotational state (both in $\mathrm{X}^{1} \Sigma^{+}$ground-state). For the alkali-metal-atom+ alkali-metal-dimer system, these levels are additionally split by the hyperfine interactions of the atomic collision partner. The rotational splitting in the $\mathrm{SrF}$ molecule in the $\mathrm{X}^{1} \Sigma^{-}$ground-state needs additional comments. Firstly, due to non-zero electronic spin, each rotational level is split by the spin-rotation interaction into states described by $j=n+s$. Secondly, these states are split by the fluorine hyperfine interaction. Overall, there are 16 hyperfine levels in the ${ }^{88} \mathrm{SrF}$ which can be populated for $n=0$ and $n=1$ rotational states. In field free case we use $f=j+i$ quantum number to label them, accordingly.

Cold polar molecules considered in this paper exhibit a permanent dipole moment 
(PDM) in their own frame (identical for all isotopologues to a very good approximation) large enough (see, e.g. [51]) to strongly interact with a static electric field. Noticeable Stark shifts comparable to $\Delta E_{a-b}$ at zero field can be induced by applying an experimentally achievable electric field. Figures 1(a), 2(a), and 3(a) show the variation of the level energies of the reactant and product complexes with the electric field for three typical cases, ${ }^{40} \mathrm{~K}+{ }^{39} \mathrm{~K}^{23} \mathrm{Na},{ }^{87} \mathrm{Rb}+{ }^{85} \mathrm{Rb}{ }^{133} \mathrm{Cs}$, and ${ }^{88} \mathrm{Sr}+{ }^{86} \mathrm{Sr}^{19} \mathrm{~F}$ collisions, respectively. The energy levels of reactants and products are parallel, with different splittings within each $n$ manifold due to the change of hyperfine states. In case of molecules with lighter atoms, such as NaK or SrF molecules, the hyperfine splittings of the reactant atoms are much smaller than rotational energy of the molecules. On the other hand, the Rb hyperfine splitting is much larger than the RbCs rotational constant, so the pattern of thresholds of reactants and products is quite complex. Note that the hyperfine states of molecules are superimposed at the resolution of the plot.

As can be seen in the panels (a) of the figures, it is possible to induce crossings between the energies of reactants and products by tuning the electric field, thus closing or opening reaction thresholds for the related collisions. For the ${ }^{39} \mathrm{~K}+{ }^{40} \mathrm{~K}{ }^{23} \mathrm{Na}$ (Fig. 1 (a)), close to $4 \mathrm{kV} / \mathrm{cm}$, the ground state of the reactant ${ }^{23} \mathrm{Na}^{39} \mathrm{~K}$ approaches $m_{n}=0$ states of ${ }^{23} \mathrm{Na}^{40} \mathrm{~K}$ molecule (which for zero field correlate with $n=1$ ), and becomes a closed channel while the electric field is increased further. The same situation can be seen close to $7.5 \mathrm{kV} / \mathrm{cm}$ : the channels with $\left|m_{n}\right|=1$ close as the electric field is swept across the state crossing. On the other hand, there are multiple crossings of the ground state of reactants with rotationally excited states of product molecules up to $n=2$ in case of the RbCs system (Fig.2 (a)). A magnitude of electric field above $15 \mathrm{kV} / \mathrm{cm}$ closes channels corresponding to $n=2$ states of product ${ }^{87} \mathrm{Rb}^{133} \mathrm{Cs}$. The reaction starting with ${ }^{88} \mathrm{Sr}+{ }^{86} \mathrm{SrF}$ (see Fig, 3 (a)) is very peculiar, since the reactant ground-state energy is very close to the one of rotationally-excited product states, and an electric field of about $2.5 \mathrm{kV} / \mathrm{cm}$ is sufficient to close all channels except ground rotational $n=0$ state. The molecules which are produced in $n=1$ state are very slow with total translational energy on the order of $20 \mathrm{mK}$ or less.

The statistical approach for collisions was initially formulated in the case of nuclear scattering by Feshbach [52, 53, 54, and adapted for molecular collisions by Bernstein, Light, and Miller [55, 56, 57]. It has been since then extended and combined with more rigorous close-coupling schemes to study the reactive scattering in many-partial wave regime for a number of important chemical reactions [58, 59, 60]. Recently, the statistical approach was used for ultracold collision studies between $\mathrm{Li}$ and $\mathrm{LiYb}$, and compared to quantum reactive scattering [61]. Here, the statistical approach of [34] is extended to the case of ultracold atom/molecule collisions in a presence of an electric field, and is used to analyze the product state distributions in reactions with exothermicity on the order of tens to hundreds of kelvins.

An essential assumption of the statistical method is that collisions proceed via the long-lived intermediate complex, so that a full randomization of quantum state populations in the interaction region occurs. Then the reactants and products are 
uncorrelated, and the transition probability for reactant state $\alpha$ to product state $\beta$ is expressed as

$$
P_{\alpha \rightarrow \beta}^{M}=p_{\alpha}^{M} p_{\beta}^{M} / \sum_{\gamma} p_{\gamma}^{M}
$$

where $\gamma$ denotes all possible values $\beta$. The quantities $p_{\alpha}^{M}$ and $p_{\beta}^{M}$ are the probabilities for capture in the entrance channel, and isotopologue formation in the outgoing channel. All these probabilities depend on the collision energy $E$ and on the electric field magnitude $\varepsilon$. Note that in the external field the total angular momentum $J$ of the collisional complex is not conserved but its projection $M$ onto the space-fixed axis (along the direction of the electric field) is.

The statistical method should be particularly useful for collisions of heavy atoms with alkali-metal dimers and systems with monofluorides of atoms with an external $s^{2}$ shell, like alkaline-earth atoms. The method relies on complex formation due to a presence of resonances and for systems with large reduced mass, small rotational constants, and a rich hyperfine structure. The initial estimations of the density of states near the collision threshold were performed by Mayle et al. 62] and recently revisited by Christianen et al. [63] for the systems with neglected hyperfine structure. In the latter paper, the density of states which do not conserve the total angular momentum but conserve its projection (which is the case for electric field) for $\mathrm{K}_{2}+\mathrm{Rb}$ collisions was calculated to be on the order of $10^{4}$ states per inverse Kelvin which is two orders of magnitude more than in case when the total angular momentum is conserved (in fieldfree case). This number is possibly smaller for $\mathrm{NaK}+\mathrm{K}$ system but larger for $\mathrm{RbCs}+\mathrm{Rb}$. The density of states in all systems concerned here is, in fact, more significant due to complex hyperfine structure. The strongest effect which couples different hyperfine states is nuclear quadrupole interaction with the electric field gradients at nucleus which changes with geometry, but also interaction-induced modification of hyperfine coupling of alkali-metal atoms [64] or SrF molecule. The detailed analysis of resonances in these complexes in an electric field with hyperfine couplings included is very challenging. However, for the sake of the present paper, one can safely assume that statistical regime is achieved in all systems studied in the present paper.

The reaction cross-section $\sigma_{\alpha \rightarrow \beta}^{M}$ for a collision at energy $E$ (taken from an arbitrary origin) and an electric field magnitude $\varepsilon$ can be expressed as

$$
\sigma_{\alpha \rightarrow \beta}^{M}(E, \varepsilon)=\frac{\pi \hbar^{2}}{2 \mu\left(E-E_{\alpha}(\varepsilon)\right)} \sum_{M} \frac{p_{\alpha}^{M}(E, \varepsilon) p_{\beta}^{M}(E, \varepsilon)}{\sum_{\gamma} p_{\gamma}^{M}(E, \varepsilon)}
$$

where $\mu$ is the reduced mass of the reactants, $E-E_{\alpha}(\varepsilon)$ gives the effective collisional energy above the reactant energy $E_{\alpha}$ at a given $\varepsilon$. In the studied cases, the initial channel corresponds to a single hyperfine state of the reactant molecule and of the atom partner, colliding in the $s$-wave regime (i.e., the $\ell$ quantum number associated to the atom-molecule mutual rotation is equal to 0 , and it is assumed to be decoupled from the other angular momenta). Following [34], we obtained the energy levels of molecules concerned by diagonalizing the Hamiltonian of the 
system including the hyperfine structure and the Stark operator (see e.g. [65, 66]). We used accurate atomic and molecular constants to obtain the energy levels of the systems [46, 49, 50, 67, 37, 45, 11, 38, 68, 36, 39, 40, 48, 69, 70, 65, 66, 41, 35, 47. Although the spacing between hyperfine energy levels of ${ }^{1} \Sigma^{+}$molecules is several orders of magnitude smaller than the one between rotational levels, we included them to properly account for state counting. To calculate complex formation probability $P_{\alpha, \beta}^{M}$, we used the semi-classical approach of tunneling through the reaction barrier (which in this case reduces to the centrifugal barrier): unit probability for $E-E_{\gamma}$ above the centrifugal barrier (similarly as in Langevin model), and a non-zero probability of the tunneling effect expressed in the Wentzel-Kramers-Brillouin model (WKB) otherwise, which can be written as [71, 34]:

$$
p_{\gamma}^{M}(E, \varepsilon)=\exp \left\{-\frac{2}{\hbar} \int_{R_{\min }}^{R_{\max }} \sqrt{2 \mu_{\gamma}\left[\frac{\ell_{\gamma}\left(\ell_{\gamma}+1\right) \hbar^{2}}{2 \mu_{\gamma} R^{2}}-\frac{C_{6}}{R^{6}}-\left(E-E_{\gamma}(\varepsilon)\right)\right]} d R\right\},
$$

where the $R_{\min }$ and $R_{\max }$ are classical turning points characterizing a position of the centrifugal barrier and $C_{6}$ is the long-range dispersion coefficient. We took van der Waals $C_{6}$ coefficients from Ref. [69] to represent long-range potentials.

Figures $1 \mathrm{~b}, 2 \mathrm{~b}$, and $3 \mathrm{~b}$ show the probabilities of forming the atom+diatom products with the specific translational energy for the reactants with an initial collisional energy of $1 \mu \mathrm{K}$, while the panel (c) therein shows the formation probability of the molecules in a given $\left|m_{n}\right|$ quantum state. Each curve in the panels (c) is a sum of all contributions to the probability presented in the panels (b). Interestingly, for zero electric field, the largest propensity of forming the product molecule corresponds to the state with the largest energy difference between ground state $n=0$ and product state. That is due to the distribution of products among all accessible $\ell$ quantum numbers in an outgoing channel is the largest. This result can be rationalized using the phasespace theory (PST) of Pechukas and Light [56], which is essentially a particular case of Eq. 3 in which step-function probabilities (equal to 0 for barrier reflection, and equal to 1, for transmission over the barrier) are used. If we neglect the hyperfine states, one can easily show that for final rotational state $n$ of products, one should populate $(2 n+1)\left(\ell_{\max }(n)+1\right) /\left(\sum_{n \leq n_{\max }}(2 n+1)\left(\ell_{\max }(n)+1\right)\right)$, where $\ell_{\max }(n)$ is the maximum end-over-end angular momentum for which the centrifugal barrier fits below $\Delta E_{a-b}-B_{0} n(n+1)$. In Table 1 , we specified the largest possible $\ell_{\max }$ value corresponding to $n=0$ in the product state. These values are on the order of 20 , so clearly, in the outgoing channel, we are no longer in the quantum regime. Approximate estimation of branching ratios in the zero-field with the PST theory agree well with calculations using the theory developed in Ref. [34] for $\mathrm{K}+\mathrm{NaK}$ and $\mathrm{Sr}+\mathrm{SrF}$ reactions, for which the energy level splitting due to hyperfine coupling is much smaller than the rotational spacing.

An inspection of Figure $1 \mathrm{~b}$ reveals that the zero-field probabilities for the $\mathrm{K}+\mathrm{NaK}$ system are $63 \%$ for the channels corresponding to $n=1$ state with a translational energy order of $100 \mathrm{mK}$ and $37 \%$ for $n=0$ state with energy about $400 \mathrm{mK}$. Interestingly, 
product distributions for the $\mathrm{K}+\mathrm{NaK}$ system are less complicated than in case of the $\mathrm{Rb}+\mathrm{RbCs}$ reaction(see Figure $2 \mathrm{~b}$ ). For the latter one, probabilities are $22 \%$ for $60 \mathrm{mK}$, and $34 \%$ for $110 \mathrm{mK}$ in a mixture of products with $n=1$, and $n=2$ states, and $26 \%$ for separated $n=1$ state with $210 \mathrm{mK}$, and $9 \%$ for $n=0$ states with energy about 100 $\mathrm{mK}$, and $250 \mathrm{mK}$. In case of the $\mathrm{Sr}+\mathrm{SrF}$ reaction, probabilities are $60 \%$ for $n=0$ state with an energy order of $750 \mathrm{mK}$, and $40 \%$ for energies between about $10 \mathrm{mK}$, and 20 $\mathrm{mK}$ which correspond to $n=1$, and $n=0$ states, respectively.

Reaction products and their probability distributions can be controlled by tuning of an external electric field, since the energy levels have different effective dipole moments (defined as energy derivative with respect to electric field). The Stark shifts of the rotational energy of reactants close reactive channels if product states are above reactant states in the energy. As the energy gap between reactant and product channel changes the number of possible $L_{\max }$ states, which can cross the centrifugal barrier decreases. Hence the number of product states also decreases with the energy gap, as the $L_{\max } \approx\left(\sqrt{108} \Delta E / E_{\mathrm{vdw}}\right)^{\frac{1}{3}}$. For this reason it is rather inefficient to tune the difference between products and reactants arbitrarily close with the electric field as a very small quantity of very cold products will be produced in such reaction. For the ${ }^{40} \mathrm{~K}(\mathrm{~F}=9 / 2)+$ ${ }^{23} \mathrm{Na}^{39} \mathrm{~K}(\mathrm{v}=0, \mathrm{n}=0)$ reaction, the intensity of the electric field of $8.16 \mathrm{kV} / \mathrm{cm}$ is sufficient to produce molecules in the pure rotational ground state. For a slightly detuned value of the electric field, e.g., for the intensity of about $7.3 \mathrm{kV} / \mathrm{cm}$, one can produce the molecules in the $\left|m_{n}\right|=1$ state with translational energy of products equaled $16 \mathrm{mK}$ with a probability of $10 \%$. Translational energy of products may even be smaller than $100 \mu \mathrm{K}$ for about $8.1 \mathrm{kV} / \mathrm{cm}$ but with a population of about $3 \%$. Interestingly, the probability is $23 \%$ for molecules in $n=1$ state with translational energy about 10 $\mu \mathrm{K}$ in the $\mathrm{Rb}+\mathrm{RbCs}$ system. In this reaction, the Stark shifts close reactive channels corresponded to $n=1$ state for intensities above $36.3 \mathrm{kV} / \mathrm{cm}$. The probability for production of samples of ${ }^{8} \mathrm{SrF}$ molecules with the translational energy smaller than 100 $\mu \mathrm{K}$ is $4 \%$ for an electric field $2.6 \mathrm{kV} / \mathrm{cm}$ which is a slightly detuned value of the field at which all reactive channels close, except ones for the ground rotational state.

In summary, we have shown that products of the isotopic substitution reactions are cold according to their translational energy below hundreds of $\mathrm{mK}$, and molecular products may occupy the lowest rotational states. We have also discussed the possibility of controlling the chemical reactions by the electric field in ultracold mixtures of molecules and atoms with low kinetic energy release, where one of the constituent atoms of colliding molecule is replaced by its isotope. Such experiments could be implemented with present experimental techniques, using a modest electric field (up to $20 \mathrm{kV} / \mathrm{cm}$ ). With such an experimental setup it is possible to trap a large amount of the product molecules in the trap, if the external microwave cavity trap is used, or - in case of ${ }^{2} \Sigma^{-}$ molecules - in a magnetic trap. This can open new avenues in investigating the branching ratios of chemical reactions in ultracold conditions. Given that full quantum dynamics calculations in external fields are very challenging and at present approximations need to be introduced, such experiments can shed new light on untangling complicated dynamics 


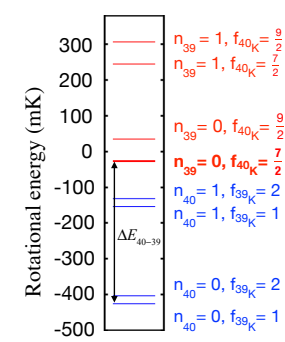

(a) Field-free levels
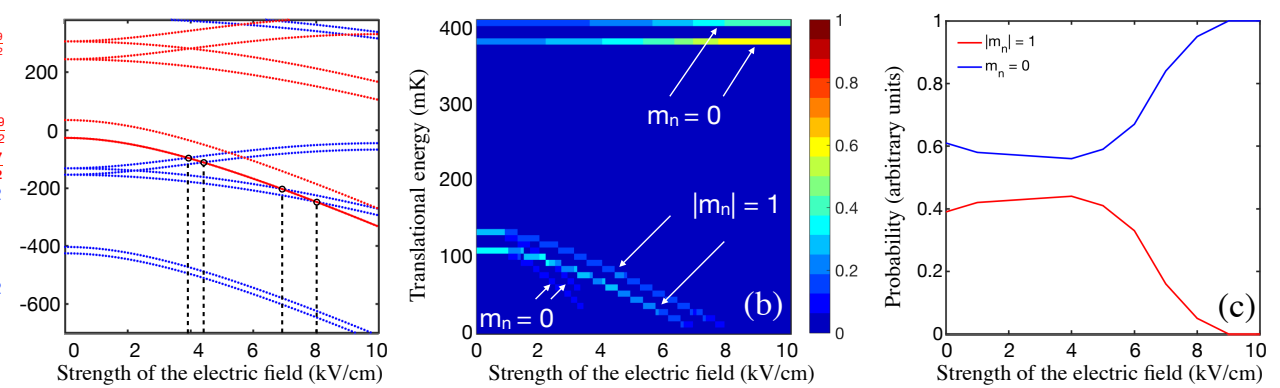

Figure 1. The ${ }^{40} \mathrm{~K}+{ }^{23} \mathrm{Na}^{39} \mathrm{~K} \longrightarrow{ }^{39} \mathrm{~K}+{ }^{23} \mathrm{Na}^{40} \mathrm{~K}$ reaction. (a) Splitting of the molecular rotational levels in a absence and presence of external electric field. The diagram shows field-free rotational levels which are labeled by $n_{39}$ (indicating ${ }^{23} \mathrm{Na}^{39} \mathrm{~K}$ ) and $n_{40}$ (indicating ${ }^{23} \mathrm{Na}^{40} \mathrm{~K}$ ) quantum numbers as well as by $f=i+s$ quantum number describing the hyperfine interactions of the atomic partner $\left({ }^{40} \mathrm{~K}\right.$ for reactants and ${ }^{39} \mathrm{~K}$ for products). The plot shows a splitting of these levels due to the interaction with external electric field. Note that, the hyperfine states of the system are superimposed the resolution of the diagram and plot. The $\Delta E_{40-39}$ is the reaction exothermicity. The red thick line corresponds to initial state of the reactants. The dashed vertical lines in the plot indicate crossings between reactant and product states due to the Stark effect. (b) Product distribution of the translational energy. (c) Distribution of the projection of the rotational quantum number, $m_{n}$, corresponding to ${ }^{23} \mathrm{Na}^{40} \mathrm{~K}$ molecule. The results presented in panels (b) and (c) are obtained with an initial collisional energy of $1 \mu \mathrm{K}$.

near the reaction thresholds.

This research has been financed from the funds of the Polish National Science Centre (grants nos. 2017/25/B/ST4/01486 and 2017/27/N/ST4/02576). Calculations have been carried out at the Wroclaw Centre for Networking and Supercomputing (http://www.wcss.pl), Grant No. 218 (PS). This work is part of the Polonium programme.

\section{References}

[1] Ospelkaus S, Ni K K, Wang D, de Miranda M H G, Neyenhuis B, Quéméner G, Julienne P S, Bohn J L, Jin D S and Ye J 2010 Science 327 853-857 URL http://science.sciencemag. org/content/327/5967/853

[2] Ni K K, Ospelkaus S, Wang D, Quéméner G, Neyenhuis B, de Miranda M H G, Bohn J L, J Y and Jin D 2010 Nature 4641324 URL http://dx.doi.org/10.1038/nature08953

[3] de Miranda M H G, Chotia A, Neyenhuis B, Wang D, Quéméner G, Ospelkaus S, Bohn J L, Ye J and Jin D S 2011 Nat. Phys. 7 502-507 URL http://dx.doi.org/10.1038/nphys1939

[4] Ye X, Guo M, González-Martínez M L, Quéméner G and Wang D 2018 Science Advances 4 URL http://advances.sciencemag.org/content/4/1/eaaq0083

[5] Knoop S, Ferlaino F, Berninger M, Mark M, Nägerl H C, Grimm R, D'Incao J P and Esry B D 2010 Phys. Rev. Lett. 104(5) 053201 URL https://link.aps.org/doi/10.1103/PhysRevLett.104. 053201

[6] Rui J, Yang H, Liu L, Zhang D C, Liu Y X, Nan J, Chen Y A, Zhao B and Pan J W 2017 Nat. Phys. 13 699-703 URL http://dx.doi.org/10.1038/nphys4095 

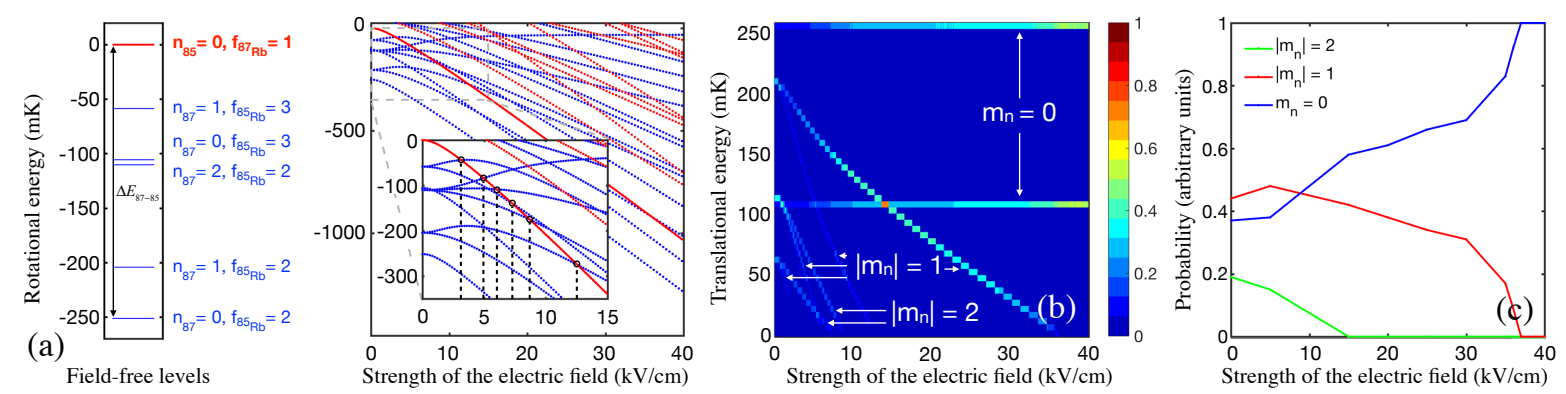

Figure 2. The ${ }^{87} \mathrm{Rb}+{ }^{85} \mathrm{Rb}^{133} \mathrm{Cs} \longrightarrow{ }^{85} \mathrm{Rb}+{ }^{87} \mathrm{Rb}^{133} \mathrm{Cs}$ reaction. (a) Splitting of the molecular rotational levels in a absence and presence of external electric field. The diagram shows field-free rotational levels which are labeled by $n_{85}$ (indicating ${ }^{85} \mathrm{Rb}^{133} \mathrm{Cs}$ ) and $n_{87}$ (indicating ${ }^{87} \mathrm{Rb}^{133} \mathrm{Cs}$ ) quantum numbers as well as by $f=i+s$ quantum number describing the hyperfine interactions of the atomic partner $\left({ }^{87} \mathrm{Rb}\right.$ for reactants and ${ }^{85} \mathrm{Rb}$ for products). The plot shows a splitting of these levels due to the interaction with external electric field. Note that, the hyperfine states of the system are superimposed the resolution of the diagram and plot. The $\Delta E_{87-85}$ is the reaction exothermicity. The red thick line corresponds to initial state of the reactants. The dashed vertical lines in the plot indicate crossings between reactant and product states due to the Stark effect. (b) Product distribution of the translational energy. (c) Distribution of the projection of the rotational quantum number, $m_{n}$, corresponding to ${ }^{87} \mathrm{Rb}^{133} \mathrm{Cs}$ molecule. The results presented in panels (b) and (c) are obtained with an initial collisional energy of $1 \mu \mathrm{K}$.
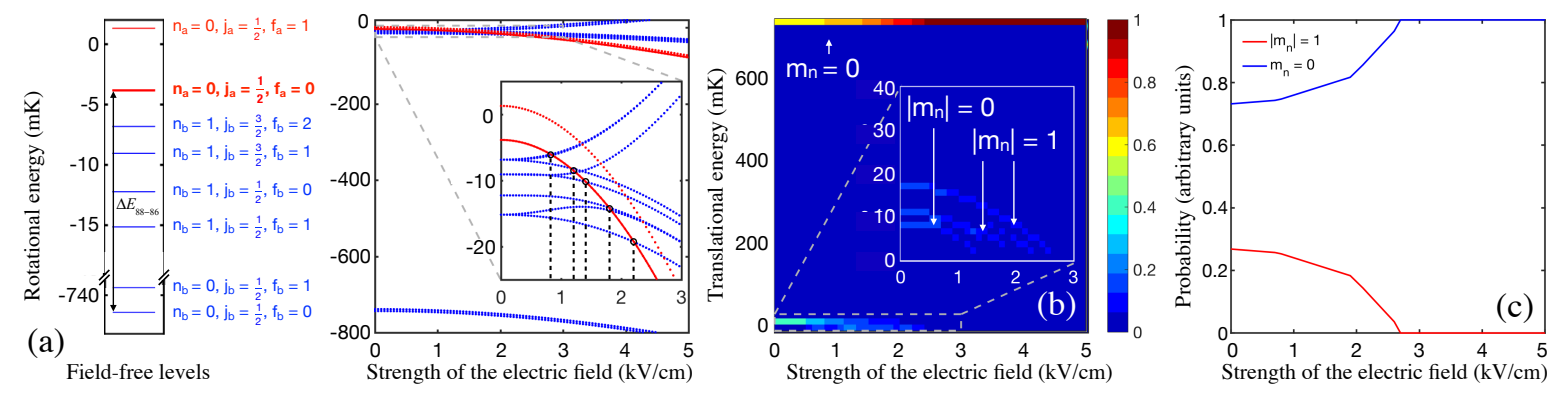

Figure 3. The ${ }^{88} \mathrm{Sr}+{ }^{86} \mathrm{Sr}^{19} \mathrm{~F} \longrightarrow{ }^{86} \mathrm{Sr}+{ }^{88} \mathrm{Sr}^{19} \mathrm{~F}$ reaction. (a) Splitting of the molecular rotational levels in a absence and presence of external electric field. The diagram shows field-free rotational levels which are labeled by the $n, j=n+s$, and $f=n+s+i$ quantum numbers which characterize to rotation, spin-rotation and hyperfine couplings in the SrF molecule. The subscripts $a$ and $b$ indicates reactants and products, respectively. The plot shows a splitting of these levels due to the interaction with external electric field. Note that, the hyperfine states of the system are superimposed the resolution of the diagram and plot. The $\Delta E_{a-b}$ is the reaction exothermicity. The red thick line corresponds to initial state of the reactants. The dashed vertical lines in the plot indicate crossings between reactant and product states due to the Stark effect. (b) Product distribution of the translational energy. (c) Distribution of the projection of the rotational quantum number, $m_{n}$, corresponding to ${ }^{88} \mathrm{Sr}^{19} \mathrm{~F}$ molecule. The results presented in panels (b) and (c) are obtained with an initial collisional energy of $1 \mu \mathrm{K}$. 
[7] Żuchowski P S and Hutson J M 2010 Phys. Rev. A 81060703 URL https://link.aps.org/doi/ 10.1103/PhysRevA.81.060703

[8] Kosicki M B, Kędziera D and Żuchowski P S 2017 J. Phys. Chem. A 121 4152-4159 URL http://dx.doi.org/10.1021/acs.jpca.7b01523

[9] Park J W, Will S A and Zwierlein M W 2015 Phys. Rev. Lett. 114205302 URL http: //link.aps.org/doi/10.1103/PhysRevLett.114.205302

[10] Will S A, Park J W, Yan Z Z, Loh H and Zwierlein M W 2016 Phys. Rev. Lett. 116(22) 225306 URL https://link.aps .org/doi/10.1103/PhysRevLett.116.225306

[11] Guo M, Zhu B, Lu B, Ye X, Wang F, Vexiau R, Bouloufa-Maafa N, Quéméner G, Dulieu O and Wang D 2016 Phys. Rev. Lett. 116(20) 205303 URL http://link.aps.org/doi/10.1103/ PhysRevLett.116.205303

[12] Molony P K, Gregory P D, Ji Z, Lu B, Köppinger M P, Le Sueur C R, Blackley C L, Hutson J M and Cornish S L 2014 Phys. Rev. Lett. 113255301 URL http://link.aps.org/doi/10.1103/ PhysRevLett.113.255301

[13] Takekoshi T, Reichsöllner L, Schindewolf A, Hutson J M, Le Sueur C R, Dulieu O, Ferlaino F, Grimm R and Nägerl H C 2014 Phys. Rev. Lett. 113205301 URL http://link.aps.org/doi/ 10.1103/PhysRevLett.113.205301

[14] Truppe S, Williams H J, Hambach M, Caldwell L, Fitch N J, Hinds E A, Sauer B E and Tarbutt M R 2017 Nat. Phys. 1311731176 URL https://www.nature.com/articles/nphys4241

[15] Williams H J, Caldwell L, Fitch N J, Truppe S, Rodewald J, Hinds E A, Sauer B E and Tarbutt M R 2018 Phys. Rev. Lett. 120(16) 163201 URL https://link.aps.org/doi/10. 1103/PhysRevLett.120.163201

[16] McCarron D J, Steinecker M H, Zhu Y and DeMille D 2018 Phys. Rev. Lett. 121(1) 013202 URL https://link.aps.org/doi/10.1103/PhysRevLett.121.013202

[17] Anderegg L, Augenbraun B L, Bao Y, Burchesky S, Cheuk L W, Ketterle W and Doyle J M 2018 Nat. Phys. 14890893 URL https://doi.org/10.1038/s41567-018-0191-z

[18] Borsalino D, Vexiau R, Aymar M, Luc-Koenig E, Dulieu O and Bouloufa-Maafa N 2016 J. Phys. B: At. Mol. Opt. Phys. 49055301 URL http://stacks.iop.org/0953-4075/49/i=5/a=055301

[19] Stevenson I C, Blasing D B, Chen Y P and Elliott D S 2016 Phys. Rev. A 94062510 URL https://link.aps.org/doi/10.1103/PhysRevA.94.062510

[20] Härter A, Krükow A, Deiß M, Drews B, Tiemann E and Denschlag J H 2013 Nat. Phys. $9512-517$ URL http://dx.doi.org/10.1038/nphys2661

[21] Wolf J, Deiß M, Krükow A, Tiemann E, Ruzic B P, Wang Y, D’Incao J P, Julienne P S and Denschlag J H 2017 Science 358 921-924 URL http://science.sciencemag.org/content/ $358 / 6365 / 921$

[22] Hu M G, Liu Y, Grimes D D, Lin Y W, Gheorghe A H, Vexiau R, Boulufa-Maafa N, Dulieu O and Ni K K 2019 arXiv:190\%.13628

[23] Tomza M 2015 Phys. Rev. Lett. 115063201

[24] Hutson J M and Soldán P 2007 Int. Rev. Phys. Chem. 26 1-28 URL https://doi.org/10.1080/ 01442350601084562

[25] Cvitaš M T, Soldán P, Hutson J M, Honvault P and Launay J M 2005 Phys. Rev. Lett. 94(3) 033201 URL https://link.aps.org/doi/10.1103/PhysRevLett.94.033201

[26] Cvitaš M T, Soldán P, Hutson J M, Honvault P and Launay J M 2005 Phys. Rev. Lett. 94(20) 200402 URL https://link.aps.org/doi/10.1103/PhysRevLett.94.200402

[27] Cvitaš M T, Soldán P, Hutson J M, Honvault P and Launay J M 2007 J. Chem. Phys 127074302 URL http://dx.doi.org/10.1063/1.2752162

[28] Quéméner G, Honvault P, Launay J M, Soldán P, Potter D E and Hutson J M 2005 Phys. Rev. A 71(3) 032722 URL https://link.aps.org/doi/10.1103/PhysRevA.71.032722

[29] Croft J F E, Makrides C, Li M, Petrov A, K K B, Balakrishnan N and Kotochigova S 2017 Universality and chaoticity in ultracold $\mathrm{k}+\mathrm{krb}$ chemical reactions URL http://dx.doi.org/ $10.1038 /$ ncomms 15897 
[30] Croft J F E, Balakrishnan N and Kendrick B K 2017 Phys. Rev. A 96(6) 062707 URL https: //link.aps.org/doi/10.1103/PhysRevA.96.062707

[31] Lara M, Jambrina P G, Launay J M and Aoiz F J 2015 Phys. Rev. A 91(3) 030701 URL https://link.aps.org/doi/10.1103/PhysRevA.91.030701

[32] Lara M, Jambrina P G, Aoiz F J and Launay J M 2015 J. Chem. Phys. 143204305 URL https://doi.org/10.1063/1.4936144

[33] Mayle M, Ruzic B P and Bohn J L 2012 Phys. Rev. A 85(6) 062712 URL https://link.aps. org/doi/10.1103/PhysRevA.85.062712

[34] González-Martínez M L, Dulieu O, Larrégaray P and Bonnet L 2014 Phys. Rev. A 90(5) 052716 URL https://link.aps.org/doi/10.1103/PhysRevA.90.052716

[35] Ivanova M, Stein A, Pashov A, Knöckel H and Tiemann E 2011 J. Chem. Phys. 134024321 URL https://doi.org/10.1063/1.3524312

[36] Adohi-Krou A, Jastrzebski W, Kowalczyk P, Stolyarov A and Ross A 2008 J. Mol. Spectrosc. 250 27-32 URL http://www.sciencedirect.com/science/article/pii/S0022285208001446

[37] Ross A J, Effantin C, Crozet P and Boursey E 1990 J. Phys. B: At. Mol. Opt. Phys. 23 L247 URL http://stacks.iop.org/0953-4075/23/i=12/a=002

[38] Ferber R, Klincare I, Nikolayeva O, Tamanis M, Knöckel H, Tiemann E and Pashov A $2008 J$. Chem. Phys. 128244316 URL http://dx.doi.org/10.1063/1.2943677

[39] Kasahara S, Ebi T, Tanimura M, Ikoma H, Matsubara K, Baba M and Katô H 1996 J. Chem. Phys. 105 1341-1347 URL http://dx.doi.org/10.1063/1.472000

[40] Fellows C E, Gutterres R F, Campos A P C, Vergès J and Amiot C 1999 J. Mol. Spectrosc. 197 19-27 URL http://www.sciencedirect.com/science/article/pii/S0022285299978803

[41] Colarusso P, Guo B, Zhang K Q, and Bernath D 1996 J. Mol. Spectr. 175 158-171

[42] Lutz J J and Hutson J M 2016 J. Mol. Spectrosc. 330 43-56 URL https://doi.org/10.1016/j. jms.2016.08.007

[43] te Velde G, Bickelhaupt F M, Baerends E J, Fonseca Guerra C, van Gisbergen S J A, Snijders J G and Ziegler T 2001 J. Comput. Chem. 22 931-967 ISSN 1096-987X URL http://dx.doi.org/ $10.1002 / j c c .1056$

[44] Perdew J P, Burke K and Ernzerhof M 1996 Phys. Rev. Lett. 77(18) 3865-3868 URL https: //link.aps.org/doi/10.1103/PhysRevLett.77.3865

[45] Russier-Antoine I, Ross A, Aubert-Frécon M, Martin F and Crozet P 2000 J. Phys. B: At. Mol. Opt. Phys. 3327532762 URL http://stacks.iop.org/0953-4075/33/i=14/a=312

[46] Arimondo E, Inguscio M and Violino P 1977 Rev. Mod. Phys. 49(1) 31-75 URL https://link. aps.org/doi/10.1103/RevModPhys.49.31

[47] Ni K K, Ospelkaus S, de Miranda M H G, Peer A, Neyenhuis B, Zirbel J J, Kotochigova S, Julienne P S, Jin D S and Ye J 2008 Science 322 231-235 URL http://science.sciencemag. org/content/322/5899/231

[48] Deiglmayr J, Aymar M, Wester R, Weidemüller M and Dulieu O 2008 J. Chem. Phys. 129064309 URL http://dx.doi.org/10.1063/1.2960624

[49] Bize S, Sortais Y, Santos M S, Mandache C, Clairon A and Salomon C 1999 Europhys. Lett. 45 558 URL http://stacks . iop.org/0295-5075/45/i=5/a=558

[50] Childs W J, Goodman G L and Goodman L S 1981 J. Mol. Spectrosc. 86 365-392 URL https://doi.org/10.1016/0022-2852(81)90288-5

[51] Aymar M and Dulieu O 2005 J. Chem. Phys. 122204302

[52] Hauser W and Feshbach H 1952 Phys. Rev. 87(2) 366-373 URL https://link.aps.org/doi/10. 1103/PhysRev.87.366

[53] Feshbach H 1958 Ann. Phys. 5357 URL https://doi.org/10.1016/0003-4916(58)90007-1

[54] Feshbach H 1962 Ann. Phys. 19287 URL https://doi.org/10.1016/0003-4916(62)90221-X]

[55] Bernstein R B, Dalgarno A, Massey H and Percival I C 1963 Proc. R. Soc. Lond. A $274427-442$ URL https://doi.org/10.1098/rspa.1963.0142

[56] Pechukas P and Light J C 1965 J. Chem. Phys. 423281 URL http://dx.doi.org/10.1063/1. 
1696411

[57] Miller W H 1970 J. Chem. Phys. 52543 URL http://dx.doi.org/10.1063/1.1673020

[58] Rackham E J, Huarte-Larranaga F and Manolopoulos D E 2001 Chem. Phys. Lett. 343 356-364 URL http://dx.doi .org/10.1016/S0009-2614(01)00707-2

[59] Rackham E J, González-Lezana T and Manolopoulos D E 2003 J. Chem. Phys. 119 12895-12907 URL http://dx.doi.org/10.1063/1.1628218

[60] González-Lezana T 2006 Int. Rev. Phys. Chem. 26 29-91 URL http://dx.doi.org/10.1080/ 03081070600933476

[61] Makrides C, Hazra J, Pradhan G B, Petrov A, Kendrick B K, González-Lezana T, Balakrishnan N and Kotochigova S 2015 Phys. Rev. A 91(1) 012708 URL https://link.aps.org/doi/10. 1103/PhysRevA.91.012708

[62] Mayle M, Quéméner G, Ruzic B P and Bohn J L 2013 Phys. Rev. A 87(1) 012709 URL https://link.aps.org/doi/10.1103/PhysRevA.87.012709

[63] Christianen A, Karman T and Groenenboom G C 2019 arXiv:1905.06691v3

[64] Barbé V, Ciamei A, Pasquiou B, Reichsöllner L, Schreck F, Żuchowski P S and Hutson J M 2018 Nat. Phys. 14881884 URL https://doi.org/10.1038/s41567-018-0169-x

[65] Aldegunde J, Rivington B A, Żuchowski P S and Hutson J M 2008 Phys. Rev. A 78(3) 033434 URL https://link.aps .org/doi/10.1103/PhysRevA .78.033434

[66] Gregory P D, Aldegunde J, Hutson J M and Cornish S L 2016 Phys. Rev. A 94(4) 041403 URL https://link.aps.org/doi/10.1103/PhysRevA.94.041403

[67] Sansonetti J E and Martin W C 2005 J. Phys. Chem. Ref. Data 341559

[68] Molony P K, Gregory P D, Ji Z, Lu B, Köppinger M P, Le Sueur C R, Blackley C L, Hutson J M and Cornish S L 2014 Phys. Rev. Lett. 113(25) 255301 URL https://link.aps.org/doi/10. 1103/PhysRevLett.113.255301

[69] Żuchowski P S, Kosicki M, Kodrycka M and Soldán P 2013 Phys. Rev. A 87(2) 022706 URL https://link.aps.org/doi/10.1103/PhysRevA.87.022706

[70] Brooks R A, Anderson C H and Ramsey N F 1972 J. Chem. Phys. 565193 URL http: //dx.doi.org/10.1063/1.1677011

[71] Balint-Kurti G G and Palov A P 2015 Theory of Molecular Collisions (The Royal Society of Chemistry) ISBN 978-1-78262-019-8

[72] Irikura K K 2007 J. Phys. Chem. Ref. Data, 36389 URL https://doi.org/10.1063/1.2436891

[73] Hessel M M 1971 Phys. Rev. Lett. 26(5) 215-218 URL https://link.aps.org/doi/10.1103/ PhysRevLett.26.215

[74] Bednarska V, Jackowska I, Jastrzȩbski W and Kowalczyk P 1998 J. Mol. Spectrosc. 189 244-248 URL https://doi.org/10.1006/jmsp.1998.7543

[75] Ridinger A, Chaudhuri S, Salez T, Fernandes D R, Bouloufa N, Dulieu O, Salomon C and Chevy F 2011 Europhys. Lett. 9633001 URL https://doi.org/10.1209/0295-5075/96/33001

[76] Staanum P, Pashov A, Knöckel H and Tiemann E 2007 Phys. Rev. A 75(4) 042513 URL https://link.aps.org/doi/10.1103/PhysRevA.75.042513 


\subsection{Supplementary material}

As mentioned in Table 1, we present complete list of the kinetic energy release, $\Delta E_{a-b}$, for all possible isotopic substitution reactions in the alkali-metal dimers. These values are obtained using the Eq. 2 . 
Isotopic substitution in ultracold molecule reactions

Table 2. The calculated reaction energy $\Delta E_{a-b}$ (references to used data in square brackets), and the rotational constant $B_{0}$, the hyperfine coupling constant $A$ of products are shown. Symbols $n_{\max }$ and $\ell_{\max }$ denote the maximum rotational state of molecule for a collision energy of $1 \mu \mathrm{K}$ and the maximum end-over-end angular momentum which can be populated by $\Delta E_{a-b}$, respectively.

\begin{tabular}{|c|c|c|c|c|c|}
\hline Products & $\Delta E_{a-b}[\mathrm{mK}]$ & $B_{0} / k_{b} T[\mathrm{mK}]$ & $A / k_{b} T[\mathrm{mK}]$ & $n_{\max }$ & $\ell_{\max }$ \\
\hline${ }^{6} \mathrm{Li}+{ }^{7} \mathrm{Li}^{23} \mathrm{Na}$ & 10747 [72] & 57073 & 73 46 & 1 & 21 \\
\hline${ }^{6} \mathrm{Li}+{ }^{7} \mathrm{Li}^{39} \mathrm{~K}$ & 97474 & 37975 & 73 46 & 1 & 22 \\
\hline${ }^{6} \mathrm{Li}+{ }^{7} \mathrm{Li}^{40} \mathrm{~K}$ & 975774 & 379 [75] & 73 46 & 1 & 22 \\
\hline${ }^{6} \mathrm{Li}+{ }^{7} \mathrm{Li}^{41} \mathrm{~K}$ & 97774 & 37975 & 7346 & 1 & 22 \\
\hline${ }^{6} \mathrm{Li}+{ }^{7} \mathrm{Li}^{85} \mathrm{Rb}$ & 96935 & 316 [48] & 7346 & 1 & 23 \\
\hline${ }^{6} \mathrm{Li}+{ }^{7} \mathrm{Li}^{87} \mathrm{Rb}$ & 969 35 & 316 [48] & 73 46 & 1 & 23 \\
\hline${ }^{6} \mathrm{Li}+{ }^{7} \mathrm{Li}^{133} \mathrm{Cs}$ & 94076 & 27948 & 73 46 & 1 & 24 \\
\hline${ }^{39} \mathrm{~K}+{ }^{6} \mathrm{Li}^{40} \mathrm{~K}$ & 27374 & 379 [75] & 1146 & 0 & 14 \\
\hline${ }^{39} \mathrm{~K}+{ }^{6} \mathrm{Li}^{41} \mathrm{~K}$ & 532 74 & 379 [75] & 1146 & 0 & 18 \\
\hline${ }^{40} \mathrm{~K}+{ }^{6} \mathrm{Li}^{41} \mathrm{~K}$ & 25974 & 37975 & -14 46 & 0 & 15 \\
\hline${ }^{39} \mathrm{~K}+{ }^{7} \mathrm{Li}^{40} \mathrm{~K}$ & 29174 & 37975 & 1146 & 0 & 15 \\
\hline${ }^{39} \mathrm{~K}+{ }^{7} \mathrm{Li}^{41} \mathrm{~K}$ & 569 [74 & 379 [75] & 1146 & 0 & 18 \\
\hline${ }^{40} \mathrm{~K}+{ }^{7} \mathrm{Li}^{41} \mathrm{~K}$ & 27774 & 37975 & -14 46 & 0 & 14 \\
\hline${ }^{39} \mathrm{~K}+{ }^{23} \mathrm{Na}^{40} \mathrm{~K}$ & 41536 & 136 [45] & 1146 & 1 & 18 \\
\hline${ }^{39} \mathrm{~K}+{ }^{23} \mathrm{Na}^{41} \mathrm{~K}$ & 81136 & 13645 & 1146 & 1 & 23 \\
\hline${ }^{40} \mathrm{~K}+{ }^{23} \mathrm{Na}^{41} \mathrm{~K}$ & 39636 & 136 45] & -1446 & 1 & 18 \\
\hline${ }^{39} \mathrm{~K}+{ }^{40} \mathrm{~K}^{85} \mathrm{Rb}$ & 47037 & 5547 & 1146 & 2 & 22 \\
\hline${ }^{39} \mathrm{~K}+{ }^{41} \mathrm{~K}^{85} \mathrm{Rb}$ & 92037 & 5547 & 1146 & 3 & 26 \\
\hline${ }^{40} \mathrm{~K}+{ }^{41} \mathrm{~K}^{85} \mathrm{Rb}$ & 45037 & 5547 & -1446 & 2 & 22 \\
\hline${ }^{39} \mathrm{~K}+{ }^{40} \mathrm{~K}^{87} \mathrm{Rb}$ & 47237 & 5547 & 11 46 & 2 & 22 \\
\hline${ }^{39} \mathrm{~K}+{ }^{41} \mathrm{~K}^{87} \mathrm{Rb}$ & 923 37 & 5547 & 1146 & 3 & 28 \\
\hline${ }^{40} \mathrm{~K}+{ }^{41} \mathrm{~K}^{87} \mathrm{Rb}$ & 45137 & 5547 & -14 46] & 2 & 22 \\
\hline${ }^{39} \mathrm{~K}+{ }^{40} \mathrm{~K}^{133} \mathrm{Cs}$ & 478 38 & 44 48 & 11 46. & 2 & 23 \\
\hline${ }^{39} \mathrm{~K}+{ }^{41} \mathrm{~K}^{133} \mathrm{Cs}$ & 93738 & 44 48 & 1146 & 4 & 29 \\
\hline${ }^{40} \mathrm{~K}+{ }^{41} \mathrm{~K}^{133} \mathrm{Cs}$ & 45838 & 44 48 & -14 46] & 2 & 23 \\
\hline${ }^{85} \mathrm{Rb}+{ }^{6} \mathrm{Li}^{87} \mathrm{Rb}$ & 11535 & 316 [48] & 49 49 & 0 & 16 \\
\hline${ }^{85} \mathrm{Rb}+{ }^{7} \mathrm{Li}^{87} \mathrm{Rb}$ & 12335 & 316 48 & 4949 & 0 & 16 \\
\hline${ }^{85} \mathrm{Rb}+{ }^{23} \mathrm{Na}^{87} \mathrm{Rb}$ & 188 39 & 100 [11 & 4949 & 0 & 20 \\
\hline${ }^{85} \mathrm{Rb}+{ }^{39} \mathrm{~K}^{87} \mathrm{Rb}$ & 198 [37] & 5547 & 4949 & 1 & 22 \\
\hline${ }^{85} \mathrm{Rb}+{ }^{40} \mathrm{~K}^{87} \mathrm{Rb}$ & 19937 & 5547 & 4949 & 1 & 22 \\
\hline${ }^{85} \mathrm{Rb}+{ }^{41} \mathrm{~K}^{87} \mathrm{Rb}$ & 20137 & 5547 & 4949 & 1 & 22 \\
\hline${ }^{85} \mathrm{Rb}+{ }^{87} \mathrm{Rb}^{133} \mathrm{Cs}$ & 253 [40] & 2350 & 49 49 & 1 & 27 \\
\hline
\end{tabular}

